\title{
Planar shock cylindrical focusing by a perfect-gas lens
}

\author{
P. E. Dimotakis and R. Samtaney ${ }^{\text {a) }}$ \\ Graduate Aeronautical Laboratories, California Institute of Technology, Pasadena, California 91125
}

(Received 15 December 2005; accepted 10 February 2006; published online 17 March 2006)

\begin{abstract}
We document a gas lensing technique that generates a converging shock wave in a two-dimensional wedge geometry. A successful design must satisfy three criteria at the contact point between the gas lens and the wedge leading edge to minimize nonlinear reflected and other wave effects. The result is a single-point solution in a multidimensional parameter space. The gas lens shape is computed using shock-polar analysis for regular refraction of the incident shock at the gas lens interface. For the range of parameters investigated, the required gas-lens interface is closely matched by an ellipse or hyperbola. Nonlinear Euler simulations confirm the analysis and that the transmitted shock is circular. As the converging transmitted shock propagates down the wedge, its shape remains nearly uniform with less than $0.1 \%$ peak departures from a perfect circular cylinder segment. Departure from the design criteria leads to converging shocks that depart from the required shape. The sensitivity to incident shock Mach number, as well as the qualitative effects of the presence of boundary layers are also discussed. (C) 2006 American Institute of Physics.
\end{abstract}

[DOI: $10.1063 / 1.2186553$ ]

Converging shocks occur in such contexts as inertial confinement fusion, ${ }^{1}$ supernova collapse, ${ }^{2}$ sonoluminescence, ${ }^{3}$ shock-wave lithotripsy, ${ }^{4}$ accelerating or maneuvering aircraft that can generate "superbooms," concave detonation-driven shocks, ${ }^{6}$ and in others. Interest in them derives from their ability to concentrate energy in a small volume, especially if the focusing is in three dimensions. Although two- and three-dimensional focusing of linear (acoustic/optical) waves is straightforward, finite-amplitude shocks exhibit instabilities that amplify initial shape imperfections ${ }^{7}$ and focusing is complicated by nonlinear wave interactions. For these and other reasons, shocks are difficult to focus in typical laboratory environments.

This Letter documents a two-dimensional (2D) gas lens that nonlinearly refracts a planar incident shock into a $2 \mathrm{D}$ circular (cylindrical) transmitted shock and focuses it as it propagates down a suitably configured wedge (Fig. 1). A properly focused shock is a prerequisite for laboratory investigations of the Richtmyer-Meshkov instability in a converging geometry, which will require the presence of a second gas interface in the converging test section. The incident planar shock, I, will, in general, refract and reflect at the gas interface contact surface, $\mathrm{C}$, which can be implemented via a suitably supported thin membrane that it will also deflect. The half-angle of the wedge, $\theta_{w}$, and the shape of $\mathrm{C}$ must generate a transmitted shock that is congruent with a circular arc centered at the wedge apex and a flow that is radial towards the wedge apex. In what follows, a subscript 0 denotes flow/gas properties ahead of the incident shock and to the left of the $\mathrm{C}$ interface, 1 denotes flow/gas properties behind the incident shock and to the left of $\mathrm{C}$, and 2 denotes conditions in the undisturbed region to the right of $\mathrm{C}$.

The parameters governing the interaction of I with $\mathrm{C}$ are:

\footnotetext{
${ }^{a)}$ Present address: Princeton Plasma Physics Laboratory, Princeton University, Princeton, NJ 08543.
}

the strength of the incident planar normal shock, given by its Mach number $\mathrm{M}_{i}$; for matched temperature and pressure across $\mathrm{C}$, the gas properties expressed as the density ratio $\eta=\rho_{2} / \rho_{0}$ across the contact interface, the ratios of specific heats $\gamma_{0}$ and $\gamma_{2}$ of the gas on the left and right of C, respectively; and the geometrical parameters: $\theta_{w}$, the angle $\alpha_{0}$ between the incident shock and the contact surface at the point $\mathrm{P}$, and the initial shape of $\mathrm{C}$. At the junction $\mathrm{P}$, the requirements for the initiation of a converging-shock flow solution are: (a) The transmitted shock must be perpendicular to the wedge face, (b) the reflected wave must be canceled to minimize nonlinear wave reflections from the wedge walls, and (c) the angle $\theta_{t}$ by which the flow turns across the transmitted shock should be such that the flow is radial immediately behind the transmitted shock - the flow-turning angle must equal the wedge half-angle, i.e., $\theta_{t}=\theta_{w}$ at $\mathrm{P}$.

Assuming $\left(T_{0}=T_{2}, p_{0}=p_{2}, \eta, \gamma_{0}\right.$, and $\left.\gamma_{2}\right)$, a gas lens, if it exists, must satisfy the three conditions and represents an inverse-design solution in the three-dimensional parameter space: $\left(\mathrm{M}_{i}, \alpha_{0}, \theta_{w}\right)$. The solution can be determined using Newton's method to define the shock polars and determine their intersections to satisfy the three criteria (details omitted in the interest of brevity). The problem differs from linearwave focusing in that the finite-amplitude wave deflects the "lens," as it interacts with it (Fig. 2), and must be such as to cancel the reflected wave at the wedge leading edge, P. No solutions exist if $\gamma_{0}=\gamma_{2}$; a hot gas to the left and the same lower-temperature gas to the right of $\mathrm{C}$ cannot lead to perfect focusing. Further, the need to cancel the reflected shock at $\mathrm{P}$ generally limits the solution to weak, or moderate-strength, incident shocks. Strong-shock refraction at a fast-slow interface typically transitions from regular refraction to irregular refraction with a Mach stem, while weak to modest shock refraction transitions from a reflected shock wave to a reflected rarefaction. ${ }^{8}$

For $\eta=1.4, \gamma_{0}=1.5$, and $\gamma_{2}=1.4$, e.g., corresponding to a 


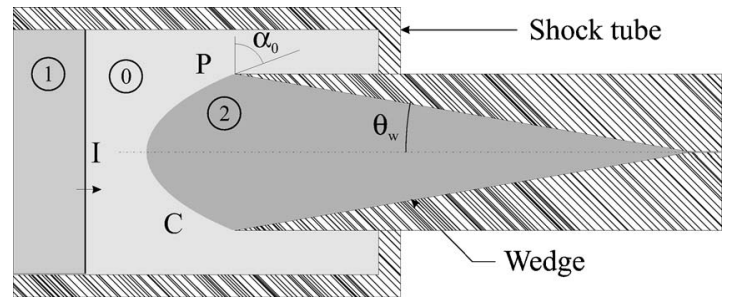

FIG. 1. Setup of the physical domain. The incident shock (I) is initialized upstream of a gas interface/contact (C).

mixture for gas 0 and nitrogen for gas 2 , the solution is $\left(\mathrm{M}_{i}=1.3122, \alpha_{0}=51.856^{\circ}, \theta_{\mathrm{w}}=11.617^{\circ}\right)$. Solutions for different gas properties, if they exist, can be found by analytic continuation.

The procedure used to compute the interface shape of $\mathrm{C}$ is now briefly described, followed by results from nonlinear Euler simulations demonstrating the correctness of the approximate analytical solution and the ability of the computed interface to focus a planar shock. Violation of any of the three confluency conditions is shown to foil the quality of the transmitted shock and the attendant focusing, where "quality" denotes the degree of congruency with a circular arc centered at the wedge apex. A sensitivity analysis in terms of incident shock strength is also presented. Finally, we comment on the influence of boundary layers in an experimental design.

The initial configuration is that of an interface with a shock upstream of it (Fig. 1). At an intermediate time, the configuration is as in Fig. 2. Assuming that the refraction is regular, all waves meet at a single node. In a small neighborhood of the node, all waves and contact surfaces are assumed to be straight, i.e., local-curvature effects are neglected. The incident shock I reaches $\mathrm{P}$ at $t=t_{i}$, having traversed the interface $\mathrm{C}$, and generates a transmitted shock $\mathrm{T}$ that must be a circular arc of radius $R$, while canceling the local reflected wave. We now solve the initial interface contour $\mathrm{C}$ that leads to the desired transmitted-shock configuration. When I refracts at $\mathrm{C}$, the transmitted shock is assumed to move in a direction normal to its front, i.e., along rays emanating from the apex of the wedge. At any time $t$, let $\alpha(t)$ be the angle between the I and $\mathrm{C}$, and let the transmitted shock front make an angle $\beta(t)$ with respect to I. At $t=0$, we have $\beta(0)=\alpha(0)=0$, whereas at $t=t_{i}, \beta\left(t_{i}\right)=\theta_{w}$, and $\alpha\left(t_{i}\right)=\alpha_{0}$.

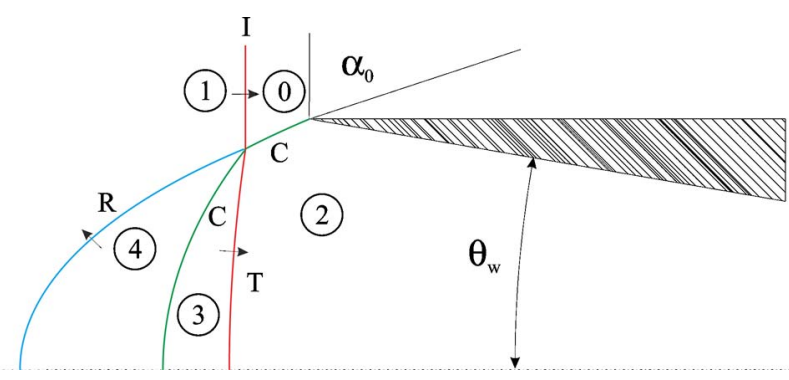

FIG. 2. (Color online) Shock-interface configuration for $t<t_{i}$, before the incident shock has traversed the interface $\mathrm{C}$. Regular refraction of the incident shock I at $\mathrm{C}$. $\mathrm{R}$ is the reflected wave and $\mathrm{T}$ is the transmitted wave that at $t_{i}$ is a circular arc centered on the wedge apex.

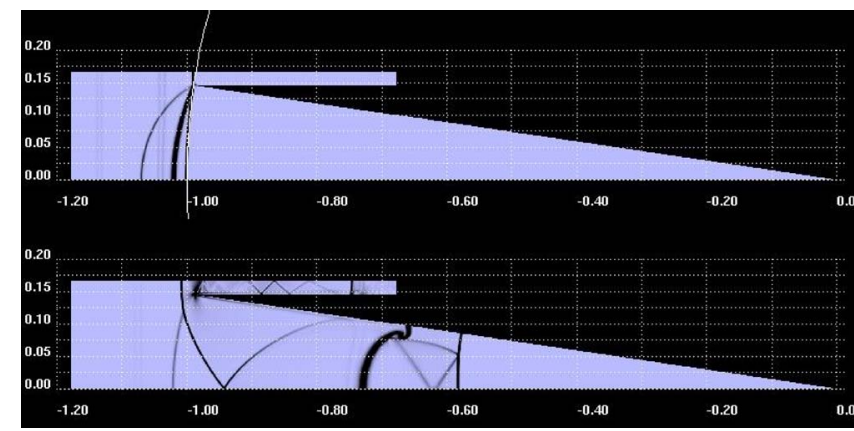

FIG. 3. (Color online) Numerical Schlieren at time $t_{i}$ (top) and at time $t$ $=6.2 t_{i}$. The white curve (top panel) is a circular arc shown for reference at time $t_{i}$. Parameters: $\mathrm{M}_{i}=3.0, \eta=1.4, \gamma_{0}=1.5, \gamma_{2}=1.4, \theta_{w}=8.37^{\circ}$, and $\alpha_{0}$ $=50.0^{\circ}$

At $t=0$, the transmitted shock strength can be estimated as a one-dimensional shock contact interaction and is $\mathrm{M}_{t 0}$ $=1.34521$ for the parameters chosen. Then, $t_{i}$ is calculated using

$$
t_{i}=\frac{R\left(1-\cos \theta_{w}\right)}{\mathrm{M}_{i} c_{0}-\mathrm{M}_{t 0} c_{2}}
$$

where $c_{0}$ and $c_{2}$ are the unshocked sound speeds in gases 0 and 2, respectively. During the time $t_{i}$, the incident and transmitted shocks travel a distance $L_{i}=\mathrm{M}_{i} c_{0} t_{i}$ and $L_{t}=\mathrm{M}_{t 0} c_{2} t_{i}$, respectively. Measuring $x$ from the wedge apex, the foot of the interface is at $x_{f}=-\left(R+L_{t}\right)$. The nonlinear function $\alpha(t)$ is then solved by an iterative bisection procedure at each time $t \in\left[0, t_{i}\right]$,

$$
\begin{aligned}
\mathcal{F}[\alpha(t)]= & \left\{R+\mathrm{M}_{t n}[\alpha(t)] c_{2}\left(t_{i}-t\right)\right\} \cos \beta(t)-\left(R+L_{t}\right) \\
& +\mathrm{M}_{i} c_{0} t=0,
\end{aligned}
$$

where $\mathrm{M}_{t n}$ is the normal Mach number of the transmitted shock. $\alpha(t)$ is the local angle between I and $\mathrm{C}$ and a function of the parameters: $\mathbf{M}_{i}, \eta, \gamma_{0}$, and $\gamma_{2} . \mathrm{M}_{t n}$ and $\beta(t)$ $\equiv \beta[\alpha(t)]$ are computed using local shock-polar analysis that, for regular refraction, as described in Ref. 9. With $\alpha(t)$ determined, the $\mathrm{C}$ interface coordinates are derived by

$$
\begin{aligned}
& x_{c}=-\left[R+\mathrm{M}_{t n} c_{2}\left(t_{i}-t\right)\right] \cos \beta(t), \\
& y_{c}=\left[R+\mathrm{M}_{t n} c_{2}\left(t_{i}-t\right)\right] \sin \beta(t) .
\end{aligned}
$$

For the design case, the required initial contact curve is (very close to) an ellipse of aspect ratio 4.448, centered at $x$ $=2.14 R$ (to the right of the wedge apex).

Results from simulations of the compressible Euler equations are now presented. The level-set method is employed to handle the wedge and "cookie-cutter" boundary geometry. Details of the numerical method are in Refs. 10 and 11. The initial conditions are a planar shock upstream of the gas interface computed in the previous section. Figure 3 shows the numerical equivalent of a Schlieren image for parameter set $\left(\mathrm{M}_{i}=3.0, \eta=1.4, \gamma_{0}=1.5, \gamma_{2}=1.4, \theta_{\mathrm{w}}=8.37^{\circ}\right.$, and $\alpha_{0}=50.0^{\circ}$ ), corresponding to a case in which the correct refracted shock is generated at $\mathrm{P}$, but the other two conditions are not satisfied. The two faint vertical lines that may be discernible are features associated with weak left-moving 


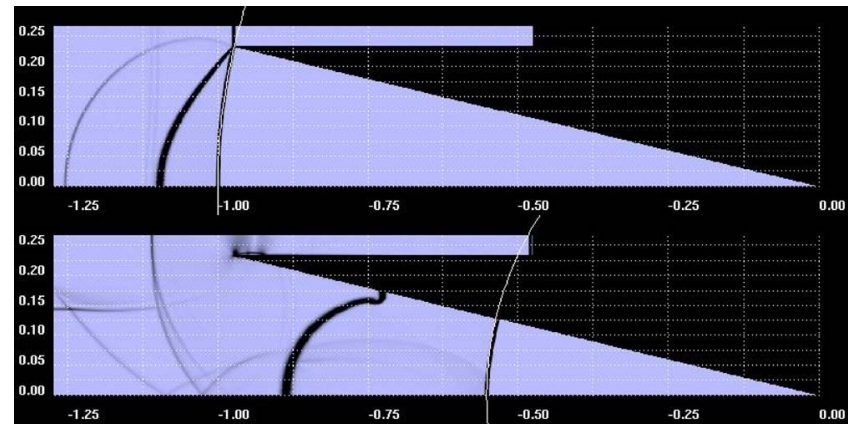

FIG. 4. (Color online) Numerical Schlieren at time $t_{i}$ (top) and at time $t$ $=3.2 t_{i}$. White circular arcs are shown for reference. Parameters: $\mathbf{M}_{i}=1.59$, $\eta=1.4, \gamma_{0}=1.4, \gamma_{2}=1.4, \theta_{w}=13.45^{\circ}$, and $\alpha_{0}=61.28^{\circ}$.

startup waves that occur as the initially sharp incident shock adjusts itself onto the discrete mesh. They are of no consequence to the subsequent development of the flow. The transmitted shock is circular at time $t_{i}$. For linear waves, this condition would suffice for focusing. However, as the finiteamplitude shock propagates into the wedge, secondary reflected waves interact with the leading shock and circularity is violated.

Figure 4 simulates a Schlieren image for the parameters: $\mathrm{M}_{i}=1.59, \quad \eta=1.4, \quad \gamma_{0}=1.4, \quad \gamma_{2}=1.4, \quad \theta_{\mathrm{w}}=13.45^{\circ}, \quad$ and $\alpha_{0}$ $=61.28^{\circ}$ ). This case satisfies the first two conditions, but not the one for radial flow behind the transmitted shock at $\mathrm{P}$, and corresponds to nearly the maximum incident Mach number for regular refraction for this combination of gases. Although the transmitted shock is circular at $t_{i}$ and the reflected shock strength vanishes at $\mathrm{P}$, circularity deteriorates with increasing time. For the $\mathbf{M}_{i}=3.0$ and $\mathrm{M}_{i}=1.59$ cases depicted in Figs. 3 and 4, respectively, the shock-polar analysis leads to an initial interface curve that closely matches a section of a hyperbola.

The design case is depicted in Fig. 5. The transmitted shock departure from circularity at $t_{i}$ is smaller than the numerical smearing from shock capturing. Reflected shock

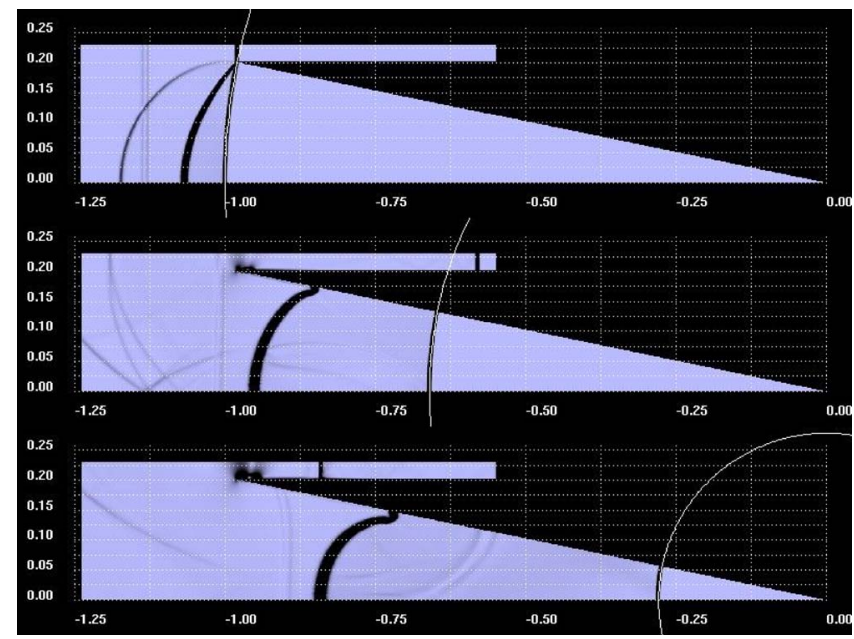

FIG. 5. (Color online) Numerical Schlieren for design case at $t=t_{i}$ (top), at $t=4.2 t_{i}$ (middle panel), and at $t=7.5 t_{i}$ (bottom panel). Solution parameters: $\mathrm{M}_{i}=1.3122, \eta=1.4, \gamma_{0}=1.5, \gamma_{2}=1.4, \theta_{w}=11.617^{\circ}$, and $\alpha_{0}=51.86^{\circ}$. White circular arcs are shown for reference.

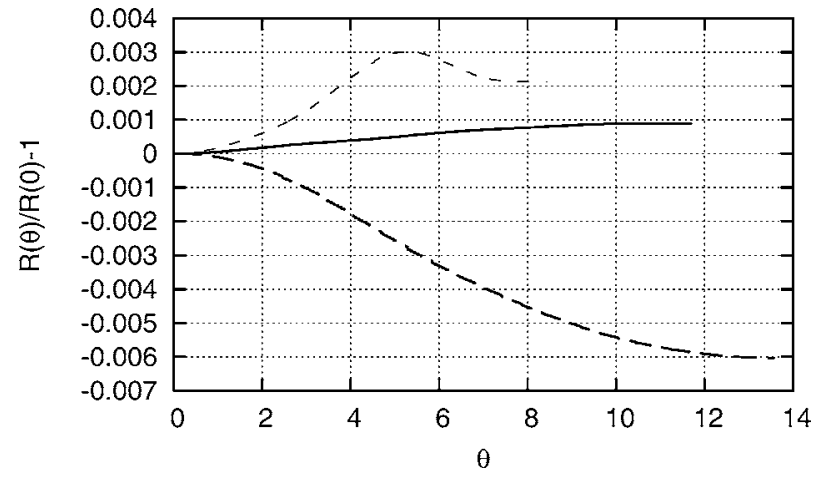

FIG. 6. Normalized shock radius, vs azimuthal angle $\theta$, for $\mathrm{M}_{i}=1.3122$ at $t=4.2 t_{i}$ (solid), $\mathbf{M}_{i}=1.59$ at $t=3.2 t_{i}$ (lower dashed), and $\mathbf{M}_{i}=3.0$ at $t=6.2 t_{i}$ (upper dashed).

strength is seen to vanish at $\mathrm{P}$ at $t_{i}$. Figure 6 depicts the normalized shock radius, some distance down the wedge, as a function of the azimuthal angle $\theta$ for the off-design and the design cases. The shocks are seen to be very nearly circular, with less than $1 \%$ deviation from a perfect circle, and less than $0.1 \%$ for the design case.

Although the design procedure yields a nearly circular transmitted shock that eventually focuses, at least for inviscid flow, the pressure behind the shock is not perfectly uniform. To leading order, the pressure behind the transmitted shock at $t_{i}$ varies as $p_{t}(\theta)-p_{t}(0)=\kappa \sin ^{2} \theta+O\left(\sin ^{4} \theta\right)$, where $\theta$ is the polar angle measured from the wedge apex, $p_{t}(0)$ is the centerline pressure behind the transmitted shock (foot of the interface), and $\kappa$ is a small parameter that depends only on $\mathrm{M}_{i}, \eta, \gamma_{0}$, and $\gamma_{2}$. As a consequence, there is a weak azimuthal flow behind the shock that will tend to spoil the desired circular symmetry. This, however, is mitigated by the fact that the converging shock accelerates as it travels down the wedge, leaving the small initial azimuthal disturbances behind. As well, the flow behind the shock is guided by the wedge (cf. results in Figs. 5 and 7).

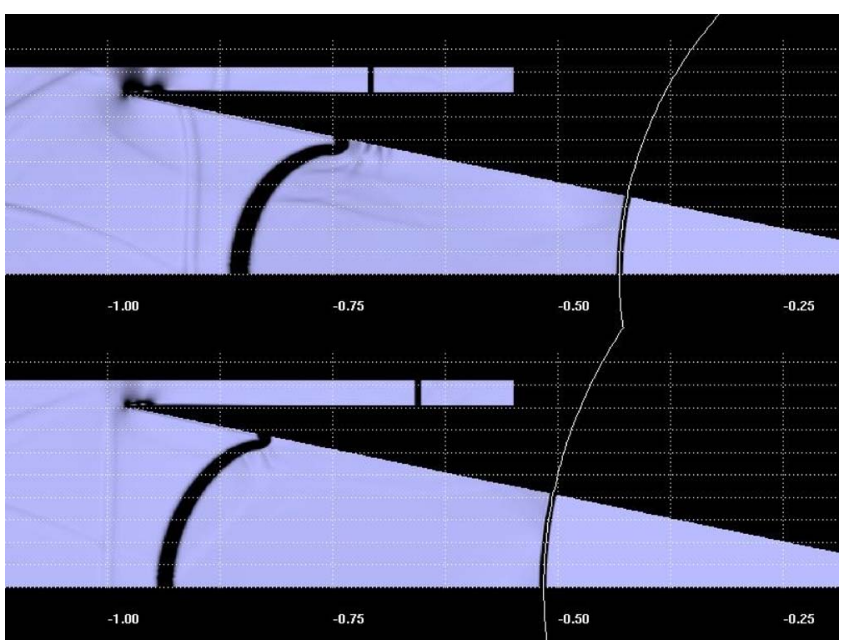

FIG. 7. (Color online) Numerical Schlieren corresponding to $\pm 5 \%$ offdesign incident Mach numbers at times. Top: $\mathrm{M}_{i}=1.3778$ at $t=5.9 t_{i}$. Bottom: $\mathrm{M}_{i}=1.2466$ at $t=5.8 t_{i}$. The white circular arc is shown for reference. 


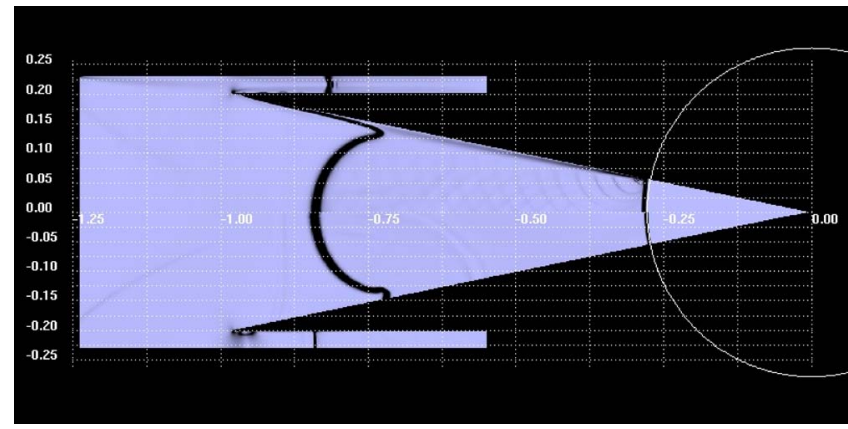

FIG. 8. (Color online) Numerical Schlieren at $t=7.5 t_{i}$. Top: design case with boundary-layer effects. Bottom: The inviscid computation shown flipped about the $x$ axis for comparison. The white circular arc is shown for reference.

In shock-tube experiments, the incident shock Mach number, $\mathrm{M}_{i}$, can be controlled to within $1-2 \%$, or so. We examine the sensitivity of the performance of the computed gas lens to $\mathrm{M}_{i}$, varying it by $\pm 5 \%$ from the optimal design value. Numerical results depicted in Fig. 7 indicate a robust focusing solution with respect to variations in $\mathrm{M}_{i}$, for the gas choices in the example considered here, for which shock polars intersect at a shallow angle.

Another consideration is the formation of boundary layers behind the shock. Their negative displacement thickness introduces flow obliquity in the vicinity of the wall, which straightens and slows the shock down as it proceeds down the wedge. In straight shock tubes, the trailing contact surface is also accelerated so, in concert with the deceleration of the shock, shock-tube test time between shock and contactsurface arrivals attains a maximum value, independent of shock-tube length. ${ }^{12,13}$ To qualitatively explore and highlight boundary layer effects in this geometry, the design case was simulated using a compressible Navier-Stokes code with noslip boundary conditions. The Reynolds number was chosen to be about two orders of magnitude lower than in planned experiments, corresponding to boundary-layer displacement effects about one order of magnitude larger than expected. No changes in the conditions at $\mathrm{P}$, or in the required design procedure for $\mathrm{C}$, are required as no boundary layers will have formed as yet. The transmitted shock at time $t_{i}$ is circular, as expected. Figure 8 compares the viscous solution (top) to the inviscid solution (bottom), at a later time. The weak waves that emanate from the shock-wave junction with the wedge wall are minor grid-induced artifacts of the numerical simu- lation. Boundary layers are responsible for some influence at later times, as expected. In particular, the converging shock with a boundary layer in its wake lags the inviscid solution at the same time. Boundary layers are also seen to have a small but discernible influence on the shape of the shock, spoiling congruency with a circular arc. These effects will be amplified further, as the ratio of the converging shock arc length to the boundary-layer displacement thickness increases with time. If the three conditions at $\mathrm{P}$ are satisfied and $\mathrm{C}$ has been designed as described previoulsy, the expected convergence ratio for shocks contained by a wedge in the laboratory will be limited by boundary layer effects.

The authors would like to acknowledge discussions with Paul Miller, Omar Hurricane, and Karnig Mikaelian of the Lawrence Livermore National Laboratory (LLNL), as well as discussions and exchanges with Hans Hornung, Dale Pullin, Dan Meiron, Amy Lam, and David Hill of Caltech. This work was performed under the auspices of the U.S. Department of Energy by the U.C. LLNL, under Contract No. W-7405-Eng-48, DOE/Caltech ASC/ASAP Subcontract No. B341492.

${ }^{1}$ D. L. Lindl, R. L. McCrory, and E. M. Campbell, "Progress toward ignition and burn propagation in inertial confinement fusion," Phys. Today 45 (9), 32 (1992).

${ }^{2}$ W. D. Arnett, J. N. Bahcall, R. P. Kirshner, and S. E. Woosley, "Supernova 1987A," Annu. Rev. Astron. Astrophys. 27, 629 (1989).

${ }^{3}$ S. J. Putterman, "Sonoluminescence-sound into light," Sci. Am. 272, 1107 (1995)

${ }^{4}$ D. Howard and B. Sturtevant, "In vitro study of the mechanical effects of shock-wave lithotripsy," Ultrasound Med. Biol. 23, 1107 (1997).

${ }^{5}$ D. J. Maglieri and K. J. Plotkin, Aeroacoustics of Flight Vehicles (Acoustical Society of America, New York, 1995).

${ }^{6}$ D. A. Holder, A. V. Smith, C. J. Barton, and D. L. Youngs, "Mix experiments using a two-dimensional convergent shock-tube," Laser Part. Beams 21, 403 (2003).

${ }^{7}$ D. W. Schwendeman and G. B. Whitham, "On converging shock waves," Proc. R. Soc. London, Ser. A 413, 297 (1987).

${ }^{8}$ A. M. Abd-El-Fattah and L. F. Henderson, "Shock waves at a fast-slow gas interface," J. Fluid Mech. 86, 15 (1978).

${ }^{9}$ R. Samtaney and N. J. Zabusky, "Circulation deposition on shockaccelerated planar and curved density-stratified interfaces: models and scaling laws," J. Fluid Mech. 269, 45 (1994).

${ }^{10}$ J. Cummings, M. Aivazis, R. Samtaney, R. Radovitzky, S. Mauch, and D. Meiron, "A virtual test facility for the simulation of dynamic response in materials," J. Supercomput. 23, 39 (2002).

${ }^{11}$ M. Arienti, P. Hung, E. Morano, and J. E. Shepherd, "A level set approach to Eulerian-Lagrangian coupling," J. Comput. Phys. 185, 213 (2003).

${ }^{12}$ A. Roshko, "On flow duration in low-pressure shock tubes," Phys. Fluids 3, 835 (1960).

${ }^{13}$ H. Mirels, "Test time in low-pressure shock tubes," Phys. Fluids 6, 1201 (1963). 